\section{Impact Time Control Based on Time-to-Go Prediction for Sea-Skimming Anti-Ship Missiles}

\author{
Min-Jea Tahk, Sang-Wook Shim, Seong-Min Hong, Chang-Hun \\ Lee and Han-Lim Choi
}

\begin{abstract}
This paper proposes a novel approach for guidance law design to satisfy the impact-time constraints for a certain class of homing missiles. The proposed guidance law provides proper lateral acceleration commands that make the impact time error converge to zero by the time of impact. This scheme can be applied to any existing guidance law for which a formula of predicted time to go is available. Convergence of time-to-go errors is supported by Lyapunov stability. The optimal guidance law (OGL) and the impact angle control guidance (IACG) law are extended by the proposed method for impact-timecontrol guidance (ITCG) and impact-time-and-angle-control guidance (ITACG), respectively. The performance of the extended guidance laws is demonstrated by numerical simulation.
\end{abstract}

Index Terms-Homing Missiles, Impact-Time-Control Guidance, Impact-Angle-Control Guidance, Time-to-Go Prediction.

\section{INTRODUCTION}

Recently advanced guidance laws have been developed by many researchers for specific mission objectives and flexibility. Among them, various impact-time-control guidance (ITCG) laws have been devised for salvo attack against naval defense systems such as close-in weapon systems in many-to-one engagement scenarios. Simultaneous attack or cooperative attack of multiple anti-ship missiles can be accomplished in two ways: by specifying the impact time of each missile or synchronizing their impact times through the communication between them. The former requires reliable ITCG laws, which has been the topic of previous studies found in [1], [2], [4]-[19].

Jeon et al. [1] have first proposed an ITCG law based on optimal control theory with linearized formulation, resulting in a guidance law which combines the conventional optimal guidance law (OGL) and a feedback loop for reducing the impact time error. In [2], Lee et al. have taken a similar approach to extend the optimal impactangle-control guidance (IACG) law [3] for impact time control. To provide additional freedom for the simultaneous control of impact angle and impact time, the jerk (time derivative of the acceleration) command is chosen as the control input. The authors of [4] have proposed a guidance law based on the differential game theory in order to control impact angle and time. Recently, Zhang et al. [5] have suggested an impact-time-and-angle-control guidance (ITACG) law which considers not only small heading/impact angles but the case of large angles. In [6], a nonlinear closed form of guidance law is introduced as quad segment polynomial trajectory guidance. The guidance law provides a minimum curvature trajectory and exact prediction of time to go.

ITCG has also been studied from the perspective of nonlinear control theory as found in [7]-[11]. Harl and Balakrishnan [7] have applied a second-order sliding mode control law for which the sliding surface is chosen to satisfy the desired line-of-sight angle profile. This is a shaping method of the line-of-sight rate to realize the desired line-of-sight angle profile, which is given as a polynomial function

This work has been supported by Agency for Defense Development (ADD) and Defense Acquisition Program Administration (DAPA) under Grant 07201-301-004.

Min-Jea Tahk, Sang-Wook Shim, Seong-Min Hong and Han-Lim Choi are with the Korea Advanced Institute of Science and Technology (KAIST), 291 Daehak-ro, Yuseong-gu, Daejeon 34141, Republic of Korea (e-mail: mjtahk, asrahiy, templersm, hanlimc@kaist.ac.kr).

Chang-Hun Lee is with the school of aerospace, transportation, and manufacturing, Cranfield University, Cranfield, Bedford, MK43 0AL, UK (e-mail: lckdgns@gmail.com). of downrange. The impact time can be determined by selecting the coefficients of the profile. In the study of Kumar and Ghose [8], sliding mode control is employed to derive an ITCG law based on time-togo estimates. The ITCG law is applicable against constant-velocity targets but impact angle constraints are not considered. Lyapunovbased ITCG laws developed by Kim et al. [9] are applicable for threedimensional engagement scenarios. The singularity issue associated with impact time control has been extensively discussed in this study. Cho et al. [10] suggest a nonsingular ITCG law composed by three components: the first term to make the time derivative of the sliding surface zero, the second term to provide Lyapunov stability, and the third term to avoid singularity. Saleem and Ratnoo [11] divide the homing guidance phase into two parts: In the first phase, the missile is injected into a straight collision course while the range is being controlled to meet the impact-time requirement. The length of the straight course is updated iteratively during the first phase.

It is noted that the Lyapunov functions used in [8]-[10] are quadratic functions of the impact time error, or precisely speaking, of the impact time error predicted on-line. This approach guarantees that the impact time error decreases monotonically if the impact time prediction is accurate. However, it does not guarantee that the impact time error converges to zero by the specified impact time. On the other hand, the guidance law proposed by [7] provides the property of finite-time convergence although the guidance law design is somewhat complicated.

Other studies on impact time control can be found in [12]-[19]. In the study of Kim et al. [12], polynomial guidance is extended to propose an ITACG law for which three coefficients of the polynomial function describing the guidance command are determined to satisfy the terminal constraints. Jung and Kim have presented an IACG law using a back-stepping method and ITCG law based on PNG in order to enhance the survivability and kill probability in [13]. An earlier work on ITACG is the study of Shin et al. [14] which adds a bias term to an IACG law to control the impact time. To accommodate the changes in the flight conditions along the path, a neural-network approximation method is proposed for real-time calculation of the bias term. In [15], a closed form of ITACG law is developed by adjusting coefficients of BPNG. In [16], an ITCG law considering seekers field-of-view constraints as well as uncertain system lag is proposed. As applications of ITCG, cooperative guidance for salvo attack is presented in [17], [18]. Recently, in [19], a vector guidance approach is proposed for a moving nonmaneuvering target. The guidance law provides a total acceleration vector which is composed of two components: perpendicular to the LOS and along the LOS. The acceleration vector is obtained by the sense of differential games to minimize a miss-distance at the desired impact time.

On the other hand, [20] and [21] have utilized geometric relationship to investigate the achievable maximum and minimum impact times in the presence of limits on maximum acceleration and fieldof-view.

This paper proposes a novel approach which utilizes a class of existing guidance laws for impact time control against stationary targets. This approach can be applied to any guidance law if a prediction formula of the time to go is provided. Specifically, the conventional optimal guidance laws, which are written in terms of the time to go, can be easily converted to an ITCG or ITACG law since reliable time-to-go prediction formulae are given for them. In this approach, a desired error dynamics of the impact time is chosen, for which the finite-time convergence of the impact time error at the target intercept can be proved through the judicious choice of the Lyapunov function as will be shown here. Thus, the impact time error is predicted from the time-to-go prediction formula, and the control input to reduce this error to zero is found from the desired 


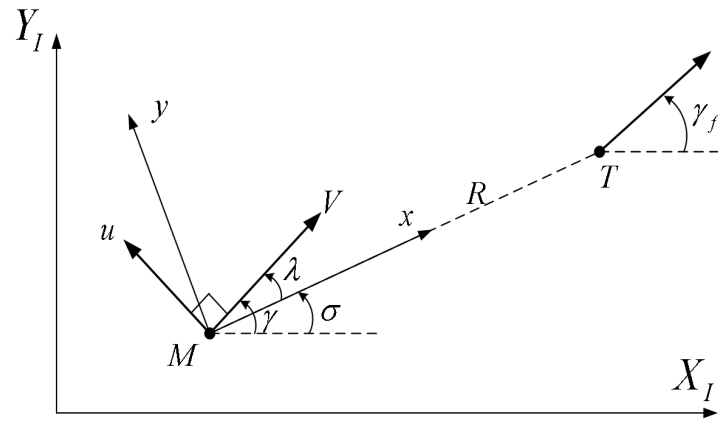

Fig. 1. Planar engagement geometry

error dynamics of the impact time. For implementation, the guidance algorithm requires the time derivative of the predicted impact time, which can be easily derived from the time-to-go prediction formula. It turns out that the resulting ITCG law is the combination of the original guidance law and an additional term for impact time control. A simple method is also proposed to circumvent inherent singularities associated with impact time control. Unless the initial condition is singular, the singularity problem can occur only for a finite number of times. Therefore, the convergence property of the proposed method is not lost as long as the lateral acceleration of the missile is not bounded. The accuracy of time-to-go prediction is important but the proposed method is found to be effective even for the case of large initial heading errors.

This paper is organized as follows: section II describes the definition of system kinematics, principal notations, and the proposed approach for impact time control. In section III, the proposed guidance law design method is applied to two optimal guidance laws, the optimal guidance law (OGL) and the optimal IACG law treated in [3], to obtain a new ITCG law and a new ITACG law, respectively. In section IV, numerical simulations are conducted with various terminal constraints to demonstrate the effectiveness of the new guidance laws.

\section{Impact Time Control Based on Time-to-go Prediction}

In this section, the system kinematics and principal notations of this paper are described, and the proposed concept for impact time control is discussed. Before introducing the system kinematics and the proposed method, we make four basic assumptions as follows:

A1. The target is stationary.

A2. The missile speed is constant.

A3. The engagement occurs in a 2-D horizontal plane.

A4. The desired impact time is achievable.

Note that these assumptions are widely accepted for guidance law design for impact time control of anti-ship missiles: (A1) If the target is moving, then the predicted position at the desired impact time can be used as a virtual target to attack. (A2) Most of sea-skimming anti-ship missiles maintain a constant cruising speed for the sake of engine control and autopilot performance. (A3) Anti-ship missile engagement against naval targets can be treated as a 2-D problem during the sea-skimming phase. (A4) To make the problem well posed, we need this assumption.

Under these assumptions, the planar engagement geometry is depicted as shown in Fig. 1, where $\left(X_{I}, Y_{I}\right)$ and $(x, y)$ represent the inertial reference frame and the line-of-sight (LOS) frame, respectively, while $\sigma$ and $\gamma$ represent the LOS angle and the missile flight path angle with respect to the inertial reference frame. The variable $\lambda$ denotes the lead angle, which is the angle between the velocity vector and the LOS. From Fig. 1, the relationships between these angles are expressed as

$$
\begin{array}{ll}
\lambda=\gamma-\sigma, & \lambda_{f}=\gamma_{f}-\sigma \\
\dot{\lambda}=\dot{\gamma}-\dot{\sigma}, & \dot{\lambda_{f}}=-\dot{\sigma}
\end{array}
$$

where the subscript $f$ represents the final time and $\gamma_{f}$ may be prescribed for impact angle control if necessary.

For a stationary target, we can obtain the engagement kinematics as follows:

$$
\begin{aligned}
\dot{R} & =-V \cos \lambda \\
\dot{\sigma} & =(-V \sin \lambda) / R \\
\dot{\gamma} & =u / V
\end{aligned}
$$

where $V, R$ and $u$ represent the missile speed, the relative distance between the missile and the target, and the lateral acceleration of the missile for maneuver, respectively. Table I explains the principal symbols used in this paper.

Hereafter, we provide the proposed idea for impact time control using existing guidance laws. Suppose that a guidance law $\Lambda$ is given with a formula for the predicted time to go, denoted as $P$. The guidance law $\Lambda$ will be extended to $\Lambda_{\text {ITC }}$ which has an additional capability of impact time control while keeping the original capabilities of $\Lambda$. Since the true time to go of $\Lambda$, denoted a $T$, is unknown, $P$ will be used for impact time control instead. Roughly speaking, $P$ is said to provide reliable time-to-go predictions if $P$ converges to $T$ as $T$ goes to 0 . In this paper, $P$ is assumed to be reliable as was in the previous impact-time-control guidance laws based on time-to-go prediction. Then, the guidance law $\Lambda$ will be modified for impact time control by making $P$ converge to $D$, which is the desired time to go.

For the guidance law $\Lambda$, the formula of predicted time to go is generally determined by the length of the missile's future trajectory over the speed (calculated in the current LOS frame) as

$$
P=\frac{\int_{0}^{R} \sqrt{1+[\gamma(x)-\sigma]^{2}} d x}{V}
$$

where the missile's future flight path angle with respect to the current LOS frame (i.e., $\gamma(x)-\sigma$ ) is assumed small. Therefore, above equation can be approximated as

$$
P \approx \frac{\int_{0}^{R}\left[1+\frac{1}{2}(\gamma(x)-\sigma)^{2}\right] d x}{V}=\frac{R}{V}(1+\eta)
$$

where

$$
\eta \triangleq \frac{1}{2 R} \int_{0}^{R}[\gamma(x)-\sigma]^{2} d x
$$

TABLE I

LIST OF SYMBOLS

\begin{tabular}{c|l}
\hline \hline Symbol & Definition \\
\hline$\sigma$ & Line-of-sight angle \\
$\gamma$ & Flight path angle \\
$\gamma_{f}$ & Flight path angle at impact \\
$\lambda$ & Lead angle \\
$t$ & Current time \\
$t_{f}$ & True impact time \\
$\hat{t}_{f}$ & Predicted impact time \\
$t_{d}$ & Desired impact time \\
$T=t_{f}-t$ & True time to go \\
$P=\hat{t}_{f}-t$ & Predicted time to go \\
$D=t_{d}-t$ & Desired time to go \\
$E=D-T$ & Impact time error \\
$\hat{E}=D-P$ & Predicted impact time error \\
\hline \hline
\end{tabular}


In Eq. (5), $\eta$ represents a parameter related with the trajectory shape characterized by the guidance law $\Lambda$. In general, $\eta$ can be obtained as a function of the LOS angle and the flight path angle. Therefore, the time-derivative of the predicted time to go is calculated as

$$
\dot{P}=\dot{\hat{t}}_{f}-1=\frac{\dot{R}}{V}(1+\eta)+\frac{R}{V}\left(\frac{\partial \eta}{\partial \sigma} \dot{\sigma}+\frac{\partial \eta}{\partial \gamma} \dot{\gamma}\right)
$$

Substituting Eq. (3) into Eq. (7) and rearranging of the result, we can express the rate of the predicted impact time as

$$
\dot{\hat{t}}_{f}=a+b u
$$

where

$$
a \triangleq 1-\cos \lambda(1+\eta)-\frac{\partial \eta}{\partial \sigma} \sin \lambda, \quad b \triangleq \frac{\partial \eta}{\partial \gamma} \frac{R}{V^{2}}
$$

Since the control input $u$ appears in Eq. (8), the predicted impact time is controllable if $b \neq 0$. To achieve the desired impact time, we need to find a proper control logic using Eq. (8). Suppose that the control input $u$ is composed of two parts as

$$
u=u_{0}+u_{1}
$$

where $u_{0}$ is the control input to keep the original predicted impact time (i.e., $\dot{\hat{t}}_{f}=0$ ) and $u_{1}$ is an additional control input to attain the desired impact time. With $u_{1}=0, u_{0}$ is determined by imposing the condition of $\dot{\hat{t}}_{f}=0$ to Eq. (8);

$$
u_{0}=-\frac{a}{b}
$$

By assumption, the predicted time to go (or the predicted impact time) given by Eq. (5) is already available for the guidance law $\Lambda$. Observe that the control input $u_{0}$ of Eq. (11) should provide the same $\hat{t}_{f}$ if $u_{1}=0$. This observation implies an interesting feature that $u_{0}$ is identical to the guidance command produced by the guidance law $\Lambda$ and produces the same characteristics of $\Lambda$. Namely, from the time-to-go prediction formula of $\Lambda$, we can readily reconstruct the original guidance law $\Lambda$ using Eq. (11).

Next, applying the control input $u$ to Eq. (8) yields

$$
\dot{\hat{t}}_{f}=b u_{1}
$$

As shown in Eq. (12), the control input $u_{1}$ is used to control the predicted impact time. Note that the impact-time-control requirement is met if $\hat{E}=D-P$, the predicted time-to-go error, is 0 when $D$ is 0 . In order for that, we suggest that the desired error dynamics of $\hat{E}$ takes the form of

$$
\dot{\hat{E}}+k\left(\frac{\hat{E}}{D}\right)=0
$$

where $k$ is some constant value. Lemma 1 provides the convergence condition of $\hat{E}$ in Eq. (13).

Lemma 1. For $k \geq 1$, the predicted impact time error $\hat{E}$ in Eq. (13) converges to zero as $D$ goes to zero and its convergence speed depends on the choice of $k$.

proof The proof is given in Appendix A.

Since the time-derivative of $\hat{E}$ is given by $\dot{\hat{E}}=-\dot{\hat{t}}_{f}$ from its definition, Eq. (13) can be rewritten as

$$
\dot{\hat{t}}_{f}=k\left(\frac{\hat{E}}{D}\right)
$$

Then, forcing the rate of the predicted impact time shown in Eq. (12) to follow the desired one given by Eq. (14), we have

$$
u_{1}=\frac{k}{b}\left(\frac{\hat{E}}{D}\right)
$$

Combining Eq. (11) and (15) provides the guidance law extended for impact time control as

$$
u=-\frac{a}{b}+\frac{k}{b}\left(\frac{\hat{E}}{D}\right)
$$

The scheme proposed above is effective if $t_{f}$ of the given guidance law can be altered properly to meet the impact-time constraint and $\hat{t}_{f}$ is a reliable prediction of $t_{f}$. In the next section, singularities associated with the control of $t_{f}$ will be discussed in details. The potential importance of the proposed method is that any existing guidance law can be modified for impact time control if it has a formula for the predicted time to go as shown in Eq. (5).

\section{Applications to Existing GUidance LaWs}

In this section, the two well-known guidance laws, OGL [1] and IACG [11], are modified by using the proposed approach for impact time control as illustrative examples.

\section{A. Extension of Optimal Guidance Law $(O G L)$}

According to reference [1], the predicted time to go of OGL is given by

$$
P=\frac{R}{V}\left(1+\frac{\lambda^{2}}{10}\right)
$$

In that case, the parameter $\eta$ is given by

$$
\eta=\frac{\lambda^{2}}{10}
$$

Then, substituting Eq. (18) into Eq. (9) gives

$$
\begin{aligned}
& a=1-\left(1+\frac{\lambda^{2}}{10}\right) \cos \lambda+\frac{\lambda}{5} \sin \lambda \\
& b=\frac{R \lambda}{5 V^{2}}
\end{aligned}
$$

Under the small angle assumption, $\sin \lambda$ and $\cos \lambda$ are approximated as $\lambda$ and $1-\lambda^{2} / 2$, respectively. Neglecting the high order term of $\lambda$, we have

$$
a=\frac{3}{5} \lambda^{2}, \quad b=\frac{R \lambda}{5 V^{2}}
$$

Then, substituting Eq. (20) into Eq. (16) provides a new guidance law for impact time control written as

$$
u=-\frac{3 V^{2}}{R} \lambda+\frac{5 k V^{2}}{R \lambda}\left(\frac{\hat{E}}{D}\right)
$$

Note that the first term of Eq. (21) is an alternative form of OGL, which is equivalent to proportional navigation guidance (PNG) with a navigation constant of 3 . The second term is an additional command to achieve the desired impact time. For convenience, the guidance law of Eq. (21) is called as ITCG from now on. As $\hat{E}$ converges to 0, the second term also goes to 0 , implying that the guidance law of (21) recovers OGL. That is, ITCG maintains the intercept performance of OGL while satisfying the impact-time requirement.

From Eq. (21), we observe that the singularity associated with impact time control occurs when $\lambda=0$. For example, if the missile is heading to the target $(\lambda=0)$ and $D>P$, the missile should turn either to the right or left. In fact, this singularity is trivial since for non-zero initial $\lambda$ it does not occur before the final time. 


\section{B. Extension of Impact-Angle-Control Guidance(IACG)}

In a similar way, the impact angle control guidance in [3] can be extended to its impact-time-control version by applying the proposed approach. The formula of the predicted time to go of IACG is given as

$$
P=\frac{R}{V}\left[1+\frac{1}{15}\left(\lambda^{2}+\lambda_{f}^{2}-\frac{1}{2} \lambda \lambda_{f}\right)\right]
$$

Therefore, the parameter regarding the trajectory curvature is given by

$$
\eta=\frac{1}{15}\left(\lambda^{2}+\lambda_{f}^{2}-\frac{1}{2} \lambda \lambda_{f}\right)
$$

Substituting Eq. (23) into Eq. (9) and imposing the small angle approximation of $\lambda$, we obtain

$$
\begin{aligned}
a= & \frac{1}{15}\left(8 \lambda^{2}-\lambda_{f}^{2}+2 \lambda \lambda_{f}\right) \\
& +\frac{1}{30}\left(\lambda^{4}+\lambda^{2} \lambda_{f}^{2}-\frac{1}{2} \lambda^{3} \lambda_{f}\right) \\
b= & \frac{R}{30 V^{2}}\left(4 \lambda-\lambda_{f}\right)
\end{aligned}
$$

Neglecting the high-order terms of Eq. (24) gives

$$
\begin{aligned}
& a=\frac{1}{15}\left(2 \lambda+\lambda_{f}\right)\left(4 \lambda-\lambda_{f}\right) \\
& b=\frac{R}{30 V^{2}}\left(4 \lambda-\lambda_{f}\right)
\end{aligned}
$$

Finally, substituting Eq. (25) into Eq. (16) provides an IACG law with the added capability of impact time control:

$$
u=-\frac{V^{2}}{R}\left(4 \lambda+2 \lambda_{f}\right)+\frac{30 V^{2}}{R\left(4 \lambda-\lambda_{f}\right)} k\left(\frac{\hat{E}}{D}\right)
$$

For convenience, we call it ITACG hereafter. From Eq. (26), the first term is an alternative form of IACG. The second term is a bias input shaping the missile's trajectory for impact time control. The bias term disappears when the predicted impact time error converges to zero (i.e., $\hat{E}=0$ ). In that case, ITACG returns to the original IACG, satisfying the impact angle constraint as well as the impact time constraint.

From Eq. (26), we observe that $u_{1}$ of Eq. (26) has $\left(4 \lambda-\lambda_{f}\right)$ in its denominator. Hence, the singularity problem associated with impact time control arises if $\lambda_{f}=4 \lambda$ holds during the flight. Suppose that the missile and the target are placed on the engagement geometry satisfying $\lambda_{f}=4 \lambda$. At this moment, the sensitivity of $\hat{t}_{f}$ to $u$ is zero and the second term of Eq. (26), $u_{1}$, goes to the infinity. However, the first term, $u_{0}$, still produces guidance commands to control the impact angle and the engagement geometry of $\lambda_{f}=4 \lambda$ will hold only momentarily. Nonetheless, the singularity produces an abrupt jump of the guidance command, which can be detrimental to the guidance performance. To circumvent this difficulty, we suggest a modified form of Eq. (26) as

$$
u=-\frac{V^{2}}{R}\left(4 \lambda+2 \lambda_{f}\right)+\frac{30 V^{2}\left(4 \lambda-\lambda_{f}\right)}{R\left[\left(4 \lambda-\lambda_{f}\right)^{2}+\delta\right]} k\left(\frac{\hat{E}}{D}\right)
$$

where $\delta>0$ is a relaxation parameter to avoid the singularity. From the comparison of Eq.s (26) and (27), we can easily show that the convergence condition of $\hat{E}$ is satisfied as long as $\left(4 \lambda-\lambda_{f}\right)^{2}(k-1)>$ $\delta$. With a small value of $\delta$, the proposed ITACG can control both the impact time and the impact angle efficiently without introducing abrupt changes of the guidance command, as will be demonstrated in the next section.

\section{Simulation Results}

In this section, the performance of the proposed guidance laws (ITCG and ITACG) is investigated through numerical simulations under various conditions. The missile is initially located at the origin of the reference frame and the stationary target is located at (10000 $\mathrm{m}, 0 \mathrm{~m})$. In the first and second subsection, a point-mass missile model with a lag-free acceleration dynamics and a maneuver limit of $10 \mathrm{G}\left(\mathrm{G}=9.8 \mathrm{~m} / \mathrm{s}^{2}\right)$ are used. It is also assumed that the missile has a constant speed of $300 \mathrm{~m} / \mathrm{s}$. In the third subsection, the performance of the proposed ITACG law is investigated in terms of robustness to the speed variations produced by the induced drag related to missile's lateral maneuvers. In this study, the lateral maneuver is assumed sustainable up to $5 \mathrm{G}$.

\section{A. Performance analysis of ITCG}

The first simulation is performed to verify the performance of ITCG with various impact time constraints $t_{d}=33.6,40$, and $50 \mathrm{sec}$. In this simulation, the initial flight path angle is fixed as $\gamma_{0}=30^{\circ}$ and the design parameter of ITCG is chosen as $k=20$.

Fig. 2a compares the missile trajectory of ITCG with that of OGL for the three impact time constraints, showing that OGL and ITCG successfully intercept the target. The impact time of OGL is recorded as $t_{f}=34.27 \mathrm{sec}$ while ITCG satisfies the impact time constraints precisely. The impact time error of ITCG turns out to be less than $0.001 \mathrm{sec}$ for the three cases of $t_{d}$. Fig. 2a clearly shows that the missile takes a longer path if a larger value of $t_{d}$ is chosen. If the designated impact time of ITCG is smaller than the impact time of OGL, the missile takes a short-cut path to meet an impact-time constraint. Fig. $2 \mathrm{~b}$ shows the lateral acceleration commands produced by the guidance laws. Obviously, the more missile maneuver is required during the initial phase for a larger $t_{d}$. For the same reason, the duration of initial acceleration saturation of $t_{d}=50 \mathrm{sec}$ is longer than that of $t_{d}=40 \mathrm{sec}$ as shown in this figure.

\section{B. Performance analysis of ITACG}

In the second simulation, the performance of the proposed ITACG law is investigated. The design parameters $k$ and $\delta$ are selected as 20 and 3, respectively. Fig. 3a shows the intercept trajectory of ITACG for $\gamma_{0}=30^{\circ}$ and $t_{d}=45 \mathrm{sec}$ with impact angles selected as $\gamma_{f}=$ $-45^{\circ}, 0^{\circ}, 45^{\circ}, 90^{\circ}$. The simulation results show that the proposed ITACG law works well even for the case of large impact angles. Fig. $3 \mathrm{~b}$ shows the time history of the flight path angle. In all cases, we observe that the proposed ITACG law successfully achieves the specified impact angle at the designated impact time. For the four cases of $\gamma_{f}$, the impact time errors are less than $0.001 \mathrm{sec}$ and the impact angle errors are less than 0.01 degree.

Fig. 4a shows the trajectory produced by the original IACG law and the proposed ITACG law for various impact times. The initial flight path angle and the designated impact angle are chosen as $\gamma_{0}=30^{\circ}$ and $\gamma_{f}=90^{\circ}$. Again, the missile takes a detour to spend time to meet the large value of designated impact time and a short-cut path for small value of designated impact time for each case of ITACG. The time history of the acceleration command is shown in Fig. 4b where we observe that ITACG needs more missile maneuvers than IACG in the initial phase for impact time control.

To study the performance sensitivity of ITACG to the design parameter $k$, the same engagement scenario is simulated with four different values of $k$ selected as $k=4,6,20$, and 40. Fig. 5a shows the trajectory of ITACG for the four different values of $k$. For all cases, ITACG provides impact time errors smaller than $0.001 \mathrm{sec}$. Also, Fig. 5b shows the history of predicted impact time error, $\hat{E}$, for each cases. According to lemma 1, the convergence speed of the 


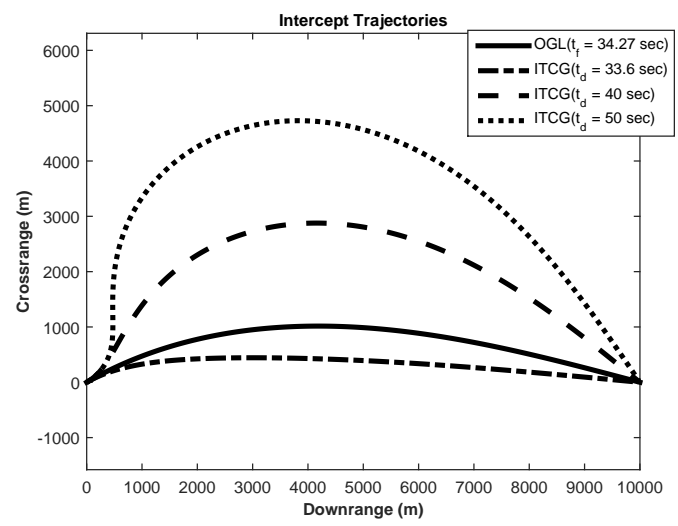

(a)

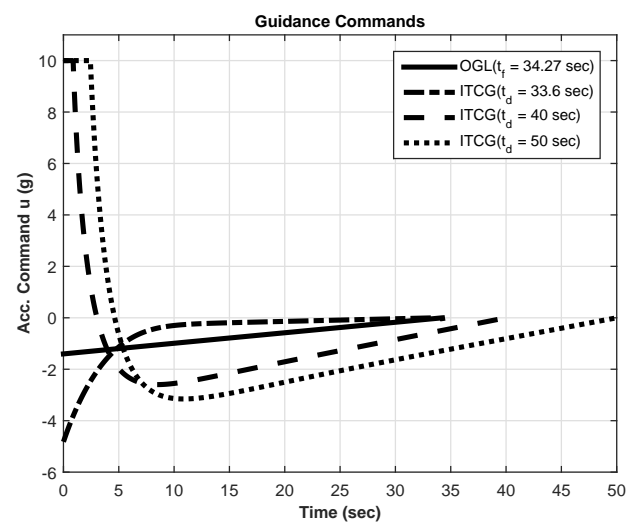

(b)

Fig. 2. Comparison of OGL and ITCG for various impact times : (a) Intercept Trajectories, (b) Guidance Commands

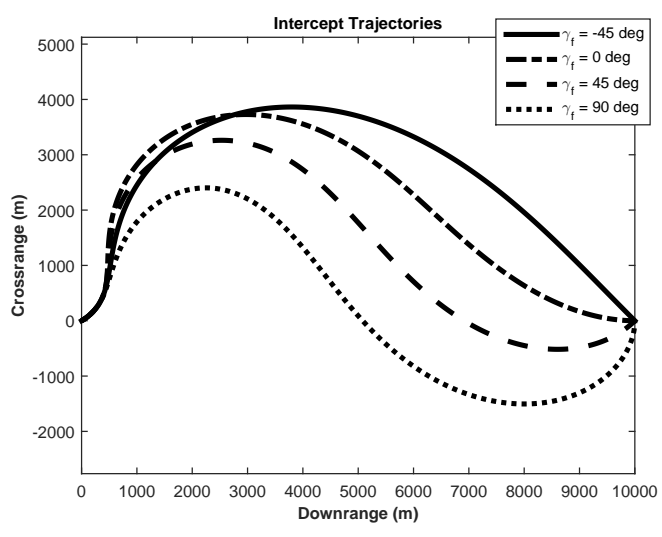

(a)

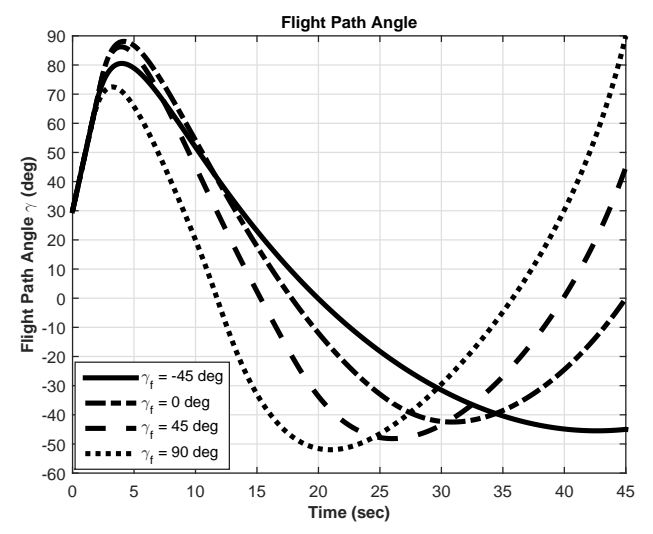

(b)

Fig. 3. Performance of ITACG for various impact angles with designated impact time of $t_{d}=45 \mathrm{sec}$ : (a) Intercept Trajectories, (b) Flight Path Angles

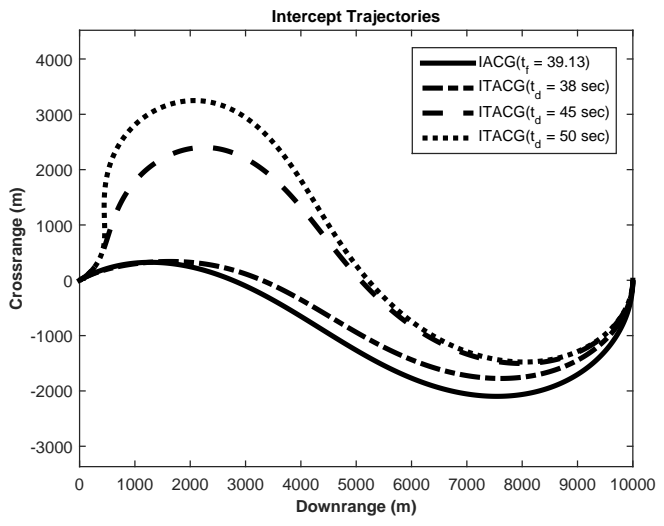

(a)

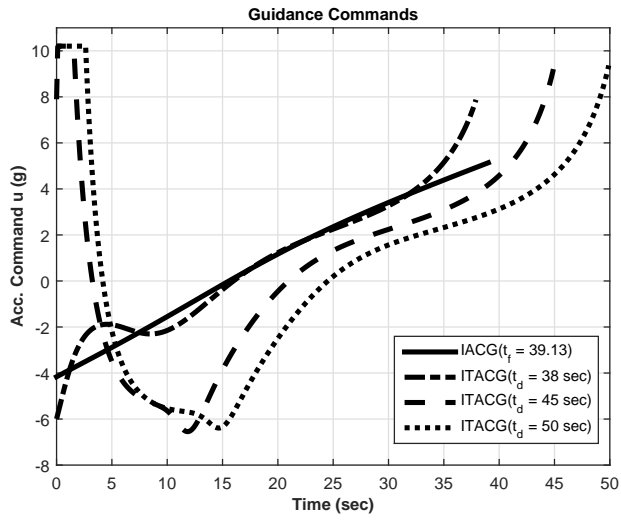

(b)

Fig. 4. Comparison of IACG and ITACG laws for various impact times with designated impact angle of $\gamma_{f}=90^{\circ}$ : (a) Intercept Trajectories, (b) Guidance Commands

impact time error increases with a larger $k$ since the correction of the impact time error occurs at the earlier stage of the engagement. The prescribed impact angle is also accurately achieved as 89.298, 89.977, 89.991 and $89.997 \mathrm{deg}$ for $k=4,6,20$, and 40, respectively. Observe that ITACG with a larger $k$ reduces the impact time error earlier, and consequently, recovers the original IACG law earlier, producing a smaller impact angle error. The simulation study indicates that the parameter $k$ can be utilized for the trade-off between the control energy during the initial phase and the impact angle accuracy. 


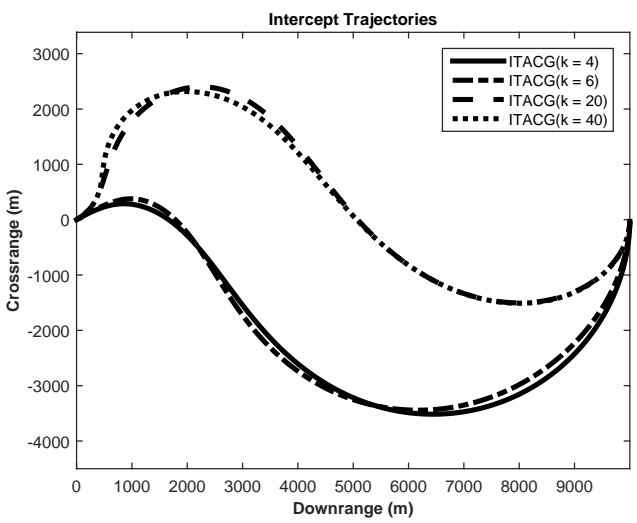

(a)

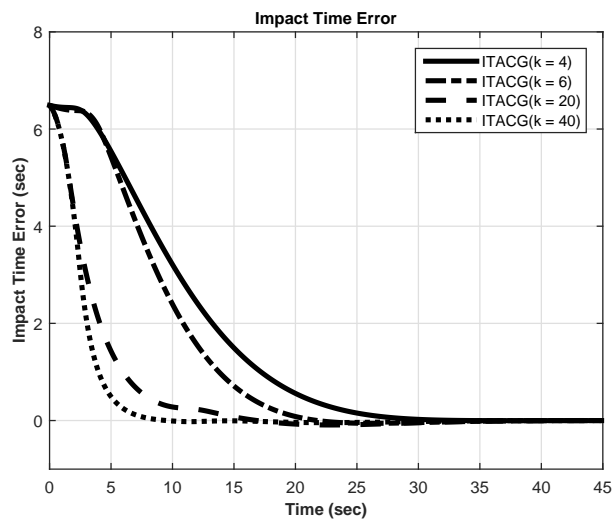

(b)

Fig. 5. Performance of ITACG with various guidance gains for designated impact time of $t_{d}=45 \mathrm{sec}$ : (a) Intercept Trajectories, (b) Predicted Impact Time Error

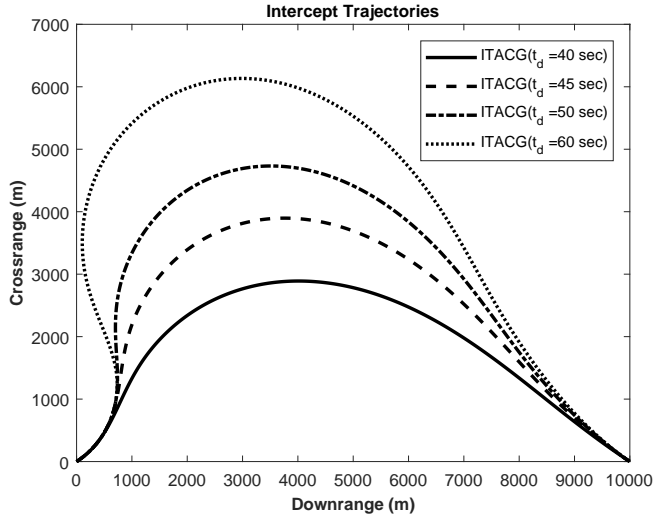

(a)

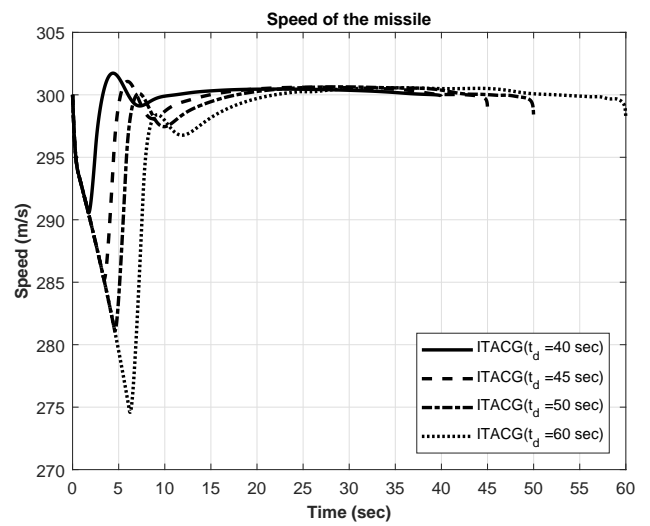

(c)

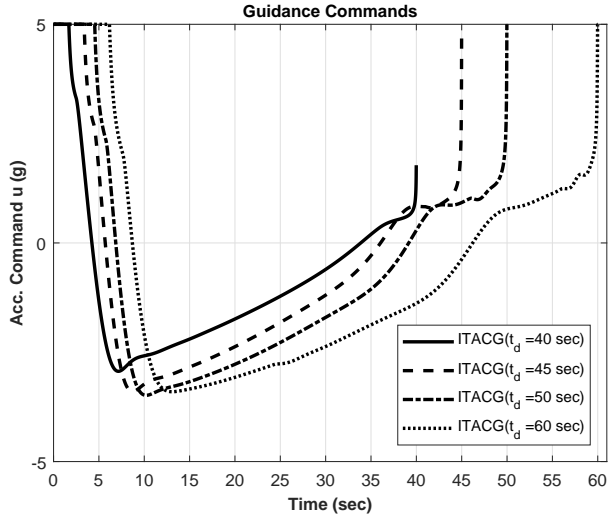

(b)

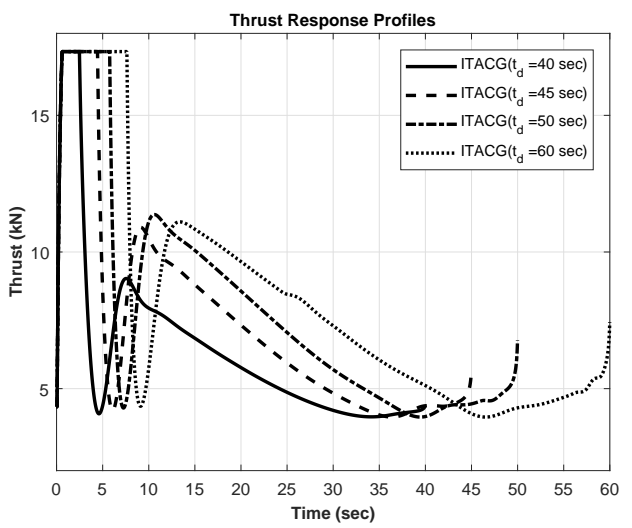

(d)

Fig. 6. Performance of ITACG with speed-varying missiles : (a) Intercept Trajectories, (b) Guidance Commands, (c) Speed Profiles, (d) Thrust Profiles

\section{Effects of missile's speed variations}

Although we assume that the anti-ship missile maintains a constant speed during the sea-skimming phase by employing thrust control, the missile experiences fluctuations in speed during lateral manuevers due to the momentary imbalance between the drag and thrust. Consider the equations of motion for the speed and the lateral acceleration written as

$$
\begin{aligned}
& \dot{V}=\frac{(F \cos \beta-D)}{m} \\
& a=\frac{Y+F \sin \beta}{m}
\end{aligned}
$$

where $F$ is the thrust, $Y$ is the lateral force, $D$ is the drag, and $\beta$ is sideslip angle of the missile. A PI-controller assumed for calculation of the thrust command, $F_{c m d}$. Then, the mathematical models for 
$F_{c m d}, Y$, and $D$ are given as

$$
\begin{aligned}
& F_{c m d}=F_{c r}+K_{p}\left(V_{\text {ref }}-V\right)+K_{I} \int\left(V_{\text {ref }}-V\right) \\
& Y=C_{Y_{\beta}} \beta q S \\
& D=\left[C_{D_{0}}+\kappa\left\{\left(C_{L_{\alpha}} \alpha_{c r}\right)^{2}+\left(C_{Y_{\beta}} \beta\right)^{2}\right\}\right] q S
\end{aligned}
$$

where $C_{D_{0}}$ is a zero-lift drag coefficient, which is modeled as constant for $M<0.8$ but linearly increasing in the Mach number range of $[0.8,0.9]$, to reflect general transonic characteristics. $C_{L_{\alpha}}$ is the lift derivative and $\alpha_{c r}$ is the trim angle of attack for seaskimming without maneuver. $C_{Y_{\beta}}$ is the lateral force derivative with respect to the sideslip angle, $\beta, \kappa$ is the induced drag correction factor, $q$ is dynamic pressure, and $S$ is the reference area. Table II lists the values of the parameters used in the simulation. In addition, the dynamic lag of the engine thrust is modeled as a 1st-order system; $F(s) / F_{c m d}(s)=K_{F} /\left(s+K_{F}\right)$. The performance of the proposed ITACG law is then investigated in terms of robustness to the speed variations produced by lateral maneuvers. The parameters of ITACG, $k$ and $\delta$, are selected as 20 and 3, respectively, and the current speed is used for the time-to-go prediction.

Fig. 6a shows the missile trajectory produced by ITACG for the desired impact angle of $\gamma_{f}=-30^{\circ}$. The initial flight path angle is $\gamma_{0}=30^{\circ}$ and the desired impact time is chosen as $t_{d}=40,45,50$ and 60 seconds, respectively. Fig. $6 \mathrm{~b}$ shows the time history of the acceleration command. The speed and thrust profiles of each case are depicted in Fig. 6c and Fig. 6d, respectively. Since the maximum thrust of the engine is limited to four times that in the cruise condition and the PI controller has a first-order system lag, the missile loses its speed rapidly right after the initiation of ITACG due to significantly large induced drag. The maximum speed drop is about $27 \mathrm{~m} / \mathrm{s}$, which is 9 percent of the cruising speed. Thereafter, the cruising speed is recovered when the required thrust is smaller than its limits, as shown in Fig. 6d. Improvement of the speed control performance is possible by adding a feedforward loop but not attempted in this study.

In spite of large speed variations in the initial phase, the simulation study shows that the desired impact times are achieved within $0.01 \mathrm{sec}$ for all cases. However, noticeable impact angle errors are observed for the cases of longer impact times. The impact angle errors are 0.06, $0.20,0.29$ and $0.27 \mathrm{deg}$ for $t_{d}=40,45,50$ and 60 , respectively. These errors can be reduced if the impact time control is turned off near the end to avoid the divergence of the lateral acceleration. A proper turn-off time could be determined by a trade-off between impact time error and impact angle error.

\section{CONCLUSION}

This paper proposes a novel approach for extending a certain class of existing homing guidance laws to satisfy the impact time constraint while keeping their original capabilities. An interesting feature of the proposed approach is that it can be applied to any homing guidance law if a reliable formula for the predicted time to go is provided. In this paper, we first reveal that the prediction formula of the time to go contains the information on how the predicted impact time changes

TABLE II

SIMULATION PARAMETERS

\begin{tabular}{c|c|c|c}
\hline \hline Symbol & value(unit) & Symbol & value(unit) \\
\hline$\kappa$ & 0.06 & $C_{L_{\alpha}}, C_{Y_{\beta}}$ & $20(-)$ \\
$C_{D_{0}}$ & From 0.4 to 0.8 & $S$ & $0.091\left(\mathrm{~m}^{2}\right)$ \\
$K_{I}$ & $400\left(\mathrm{~kg} / \mathrm{s}^{2}\right)$ & $m$ & $700(\mathrm{~kg})$ \\
$K_{P}$ & $2000(\mathrm{~kg} / \mathrm{s})$ & $V_{\text {ref }}$ & $300(\mathrm{~m} / \mathrm{s})$ \\
$K_{F}$ & $5(1 / \mathrm{s})$ & & \\
\hline \hline
\end{tabular}

when applying the control input. Based on this result, a guidance law for impact time control can be easily derived by forcing the rate of the predicted impact time to follow the desired error dynamics of the predicted impact time. Each guidance law developed by the proposed approach has two command terms: the first term, surprisingly, turns out to be the original guidance law keeping the predicted time to go constant and the second term forcing the impact time error converge to zero by the time of impact. The convergence of the impact time error under the proposed method is proved by the Lyapunov stability theory.

As illustrative examples, the two well-known optimal homing guidance laws are extended for impact time control by using the proposed approach. The singularity issue associated with impact time control is also discussed, and a remedy to avoid abrupt changes in the guidance command history is introduced. Through numerical simulations, the performance of the guidance laws based on the proposed approach is investigated. Simulation results show that the proposed guidance laws accurately meet the impact time constraints while maintaining the properties of the original guidance laws.

The proposed approach is believed to have an academic significance since it suggests a new direction for the study of impacttime-control guidance laws. Furthermore, the proposed method has a practical importance as demonstrated in the simulation study considering speed variations due to induced drag. Various new impacttime-control guidance laws are expected to be developed by applying the proposed approach to practical problems. For further study, a generalized form of impact-time-control guidance laws will be sought in the context of the approach described in this paper.

\section{APPENDIX A \\ PROOF OF LEMMA 1}

Define a Lyapunov function $W$, which is a function of $\hat{E}$ and $D$, as

$$
W=\frac{1}{2}\left(\frac{\hat{E}}{D}\right)^{2}
$$

Observe that a $D$ smaller than $T$ or $P$ does not make sense since the target cannot be reached before $t_{f}$. Therefore, $D>0$ is the domain of the interest.

It is noted that $\hat{E}$ converges to 0 if $W$ remains finite until $D$ goes to 0 . That is, $\dot{W} \leq 0$ is a sufficient condition to achieve the desired impact time $t_{d}$. Note that if $W$ is alternatively chosen as $W=\hat{E}^{2} / 2$, the finite-time convergence of $\hat{E}$ is not guaranteed even if $\dot{W}<0$. Taking the time-derivative (A.1) gives

$$
\dot{W}=\frac{\hat{E}}{D}\left(\frac{\dot{\hat{E}}}{D}-\frac{\hat{E} \dot{D}}{D^{2}}\right)
$$

Since the desired impact time, $t_{d}$, is a prescribed constant, the timederivative of the desired time to go is given as $\dot{D}=-1$. Substituting this value and Eq. (13) into Eq. (A.2) yields

$$
\dot{W}=\frac{\hat{E}^{2}(1-k)}{D^{3}}
$$

Hence, if $k \geq 1$, then $\dot{W} \leq 0$ implying that $\hat{t}_{f}$ converges to $t_{d}$ as $t$ goes to $t_{d}$.

\section{ACKNOWLEDGMENT}

This work has been supported by Agency for Defense Development (ADD) and Defense Acquisition Program Administration (DAPA) under Grant 07-201-301-004. 


\section{REFERENCES}

[1] Jeon, I., Lee, J., and Tahk, M Impact-Time-Control Guidance Law for Anti-Ship Missiles. IEEE Trans. Control Systems Technology, 14, 2 (2006), 260-266.

[2] Lee, J., Jeon I., and Tahk M. Guidance Law to Control Impact Time and Angle. IEEE Trans. Aerospace and Electronic Systems, 43, 1 (2007), 301-310.

[3] Ryoo, C., Cho, H., and Tahk, M. Optimal Guidance Laws with Terminal Impact Angle Constraint. Journal of Guidance, Control, and Dynamics, 28, 4 (2005), 724-732.

[4] Kang S., and Kim, H.

Differential Game Missile Guidance with Impact Angle and Time Constraints. Proceedings of the International Federation of Automatic Control World Congress, Milano, Italy, 2011, 3920-3925.

[5] Zhang, Y., Wang, X., and Ma, G. Impact time control guidance law with large impact angle constraint. Journal of Aerospace Engineering, /bf 229, 11 (2015), 2119-2131.

[6] Snyder, M.G., Prazenica R.J., and Hull, R.A.

A New Impact Time Control Guidance Law for Precise Time-on-Target Missile Strike. AIAA Guidance, Navigation, and Control Conference, AIAA SciTech Forum, Kissimmee, Florida, 2015.

[7] Harl, N. and Balakrishnan, S.N. Impact Time and Angle Guidance With Sliding Mode Control. IEEE Transactions on Control Systems Technology, /bf 20, 6 (2012), 1436-1449.

[8] Kumar, S.R. and Ghose, D.

Sliding Mode Control Based Guidance Law with Impact Time Constraints. American Control Conference (ACC) 2013, Washington, DC, USA, June 2013.

[9] Kim, M., Jung, B., Han, B., Lee, S., and Kim, Y.

Lyapunov based impact time control guidance laws against stationary targets. IEEE Trans. Aerospace and Electronic Systems, 51, 2 (2015), 1111-1122.

[10] Cho, D., Kim, H., and Tahk, M.

Nonsingular Sliding Mode Guidance for Impact Time Control. Journal of Guidance, Control, and Dynamics, /bf 39, 1 (2016), 61-68.

[11] Saleem, A., and Ratnoo, A.

Lyapunov based guidance law for impact time control and simultaneous arrival. Journal of Guidance, Control, and Dynamics, 39, 1 (2016), 164173.

[12] Kim, T., Lee, C., Jeon, I., and Tahk, M.

Augmented Polynomial Guidance With Impact Time and Angle Constraints. IEEE Trans. Aerospace and Electronic Systems, /bf 49, 4 (2013), 2806-2817.

[13] Jung, B., and Kim, Y.

Guidance laws for anti-ship missiles using impact angle and impact time. in AIAA Guidance, Navigation, and Control Conference, Keystone, CO, August 2006.

[14] Shin, H., Lee, J., and Tahk, M.

Guidance Synthesis to Control Impact Angle and Time. KSAS International Journal, 7, 1 (2006), 129-136.

[15] Zhang, Y., Ma, G., and Liu, A.

Guidance law with impact time and impact angle constraint. Chinese Journal of Aeronautics, /bf 26, 4 (2013), 960-966.

[16] Zhang, Y., Wang, X., and Wu, H. Impact time control guidance with field-of-view constraint accounting for uncertain system lag. Proceedings of the Institution of Mechanical Engineers, Part G: Journal of Aerospace Engineering, 230, 3 (2015), 515-529.

[17] Jeon, I., Lee, J., and Tahk, M. Homing Guidance Law for Cooperative Attack of Multiple Missiles. Journal of Guidance, Control, and Dynamics, /bf 33, 1 (2010), 275-280.

[18] Shiyu, Z., and Rui, Z.

Cooperative Guidance for Multimissile Salvo Attack. Chinese Journal of Aeronautics, /bf 21, 6 (2008), 533-539.

[19] Gutman, S.

Impact-Time Vector Guidance. Journal of Guidance, Control, and Dynamics, 40, 8 (2017), 2110-2114.

[20] Dubins, L.

On Curves of Minimal Length with a Constraint on Average Curvature, and with Prescribed Initial and Terminal Positions and Tangents. American Journal of Mathematics, 79, 3 (1957), 497-516.

[21] Sang, D., and Tahk, M.

Guidance Law Switching Logic Considering the Seeker's Field-of-View Limits. Proceedings of the Institution of Mechanical Engineers, Part $G$ : Journal of Aerospace Engineering, 223, 8 (2009), 1049-1058.

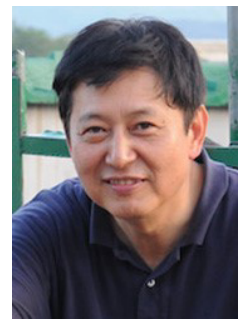

Min-Jea Tahk is a Professor of Aerospace Engineering at KAIST (Korea Advanced Institute of Science and Technology). He received the B.S. degree from Seoul National University, Seoul, Korea, and the M.S. and Ph.D. degrees from the University of Texas, Austin, in 1983 and 1986, respectively, all in aerospace engineering. From 1976 to 1981, he was a research engineer at the Agency for Defense Development in Korea, and from 1986 to 1989 he was employed by Integrated Systems, Inc., Santa Clara, CA, before he joined KAIST. His research interests are missile guidance, differential games, weapon-target assignments, and evolutionary optimization. Prof. Tahk was Technical Editor of IEEE Transactions on Aerospace and Electronic Systems from 2011 to 2016. Currently, he is Editor-in-Chief of International Journal of Aeronautical and Space Sciences.

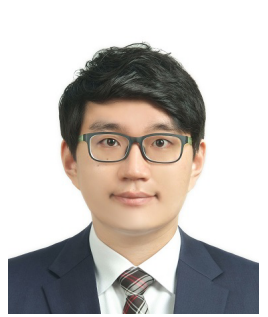

Sang-Wook Shim received the B.S., and M.S., degrees in aerospace engineering from Korea Advanced Institute of Science and Technology (KAIST), Daejeon, Korea, in 2010, and 2012, respectively. $\mathrm{He}$ is currently a $\mathrm{Ph} . \mathrm{D}$. student of Aerospace Engineering at KAIST, Daejeon, Korea. His recent research areas are advanced missile guidance and control, vision-based target detection, and target tracking filter.

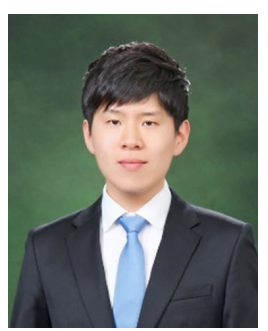

Seong-Min Hong received the B.S. degree in mechanical engineering from Sungkyunkwan University, Suwon, Korea, 2013, and the M.S. degree in aerospace engineering from Korea Advanced Institute of Science and Technology (KAIST), in 2015. $\mathrm{He}$ is currently a Ph.D. student of Aerospace Engineering at KAIST, Daejeon, Korea. His research interests include trajectory optimization and missile guidance.

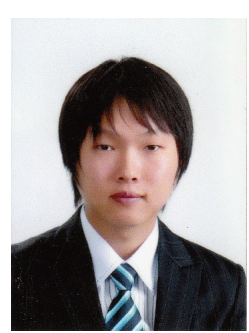

Chang-Hun Lee received the B.S., M.S., and $\mathrm{Ph} . \mathrm{D}$. degrees in aerospace engineering from Korea Advanced Institute of Science and Technology (KAIST), in 2008, 2010, and 2013, respectively. From 2013 to 2015 he was a senior researcher for guidance and control (G\&C) team at the Agency for Defense Development (ADD) in Korea. Since 2016, he has worked for School of Aerospace, Transportation, and Manufacturing at Cranfield University, UK where he is currently a research fellow. His recent research areas are advanced missile guidance and control, cooperative control for UAVs, target tracking filter, deep learning, and aviation data analytics.

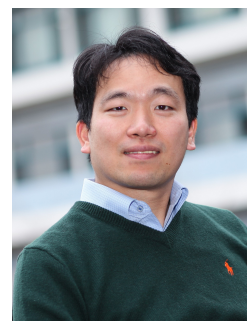

Han-Lim Choi is an Associate Professor of Aerospace Engineering at KAIST (Korea Advanced Institute of Science and Technology). He received his B.S. and M.S. degrees in Aerospace Engineering from KAIST, Daejeon, Korea, in 2000 and 2002, respectively, and his $\mathrm{PhD}$ degree in Aeronautics and Astronautics from Massachusetts Institute of Technology (MIT), Cambridge, MA, USA, in 2009. He then worked at MIT as a postdoctoral associate until he joined KAIST in 2010. His current research interests include planning and control of multi-agent systems, planning and control under uncertainty, resource management in radars, and Bayesian inference for large-scale systems. He (together with Dr. Jonathan P. How) is the recipient of Automatica Applications Prize in 2011. 
2018-02-07

\section{Impact time control based on time-to-go prediction for sea-skimming antiship missiles}

Tahk, Min-Jea

IEEE

Min-Jea Tahk, Sang-Wook Shim, Seong-Min Hong, et al., Impact time control based on time-to-go prediction for sea-skimming antiship missiles. IEEE Transactions on Aerospace and Electronic Systems, Volume 54, Issue 4, August 2018, pp. 2043-2052

https://doi.org/10.1109/TAES.2018.2803538

Downloaded from Cranfield Library Services E-Repository 